\title{
Mobilisation of enterocyte fat stores by oral glucose in humans
}

\author{
M D Robertson, M Parkes, B F Warren, D J P Ferguson, K G Jackson, D P Jewell, \\ K N Frayn
}

See end of article for authors' affiliations

Correspondence to:

Dr M D Robertson, Oxford

Lipid Metabolism Group,

OCDEM, Churchill

Hospital, Oxford OX3 7W

denise.robertson@

oxlip.ox.ac.uk

Accepted for publication 26 November 2002
Background and aims: When a high fat oral load is followed several hours later by further ingestion of nutrients, there is an early postprandial peak in plasma triacylglycerol (TG). The aim of this study was to investigate the location and release of lipid from within the gastrointestinal tract.

Methods: Ten healthy patients undergoing oesopho-gastro-duodenoscopy (OGD) were recruited. At $\mathrm{t}=0$, all patients consumed a $50 \mathrm{~g}$ fat emulsion and at $\mathrm{t}=5$ hours they consumed either water or a 38 g glucose solution. OGD was performed at $t=6$ hours and jejunal biopsy samples were evaluated for fat storage. A subgroup of five subjects then underwent a parallel metabolic study in which postprandial lipid and hormone measurements were taken during an identical two meal protocol.

Results: Following oral fat at $t=0$, samples from patients that had subsequently ingested glucose exhibited significantly less staining for lipid within the mucosa and submucosa of the jejunum than was evident in patients that had consumed only water $(p=0.028)$. There was also less lipid storage within the cytoplasm of enterocytes $(p=0.005)$ following oral glucose. During the metabolic study, oral glucose consumed five hours after oral fat resulted in a postprandial peak in plasma TG, chylomicron-TG and apolipoprotein B48 concentration compared with oral water.

Conclusion: After a fat load, fat is retained within the jejunal tissue and released into plasma following glucose ingestion, resulting in a peak in chylomicron-TG which has been implicated in the pathogenesis of atherosclerosis.
$A^{b}$ sorption of dietary lipids involves intraluminal digestion of ingested fat, transfer of the products of digestion across the brush border of enterocytes, resynthesis of the lipids, and assembly of chylomicrons. Chylomicrons are then secreted into the mesenteric lymph and transported to the thoracic duct from which they enter the circulation.

Previous studies by our group and others have documented the presence of an early peak in plasma chylomicrontriacylglycerol (TG) following successive fat rich meals. ${ }^{1-5}$ Fatty acid analysis of this early postprandial TG peak has revealed it to originate from fat contained in the previous meal ${ }^{3}$ which has led to the hypothesis of an "enteral" storage site for dietary lipids. ${ }^{136}$ However, the exact location of this storage site remains to be elucidated.

Within the enterocyte, TG is resynthesised by enzymes present on the cytosolic side of the endoplasmic reticulum (ER). Part of this resynthesised TG will enter into a secretion coupled pool inside the ER lumen, another part will potentially enter a TG storage pool located in the cytosol. ${ }^{78} \mathrm{TG}$ stored within the cytosol will only become available for lipoprotein synthesis after first undergoing lipolysis, translocation to the ER membrane, and then finally re- esterification back into TG. ${ }^{9}$ Chylomicron assembly occurs via a two step process ${ }^{10}$ which involves addition of lipid to a lipid poor apolipoprotein B48 (apo B48) particle by microsomal triglyceride transfer protein (MTP) (reviewed by White and colleagues ${ }^{11}$ ). Pre-chylomicron particles migrate to the Golgi where proteins are modified by glycosylation. ${ }^{12}$ Trans-Golgi vesicles then move to fuse with the lateral borders of the enterocyte, releasing the chylomicron into the lamina propria. ${ }^{713}$

Despite the rapid formation of TG within the enterocyte, it has been found that it takes at least four hours of constant intraduodenal lipid infusion to reach a steady state output in lymph, ${ }^{14}$ suggesting that the ability of the enterocyte to export TG is slow compared with the rate of TG synthesis. This suggests that following a meal, TG may be stored within the enterocyte. The rate of movement of TG from the ER to the Golgi correlates well with the output of TG into lymph ${ }^{14}$ and so the secretory apparatus is unlikely to be the primary site of storage. Although enterocytes are thought not to store large amounts of lipid within the cytoplasm, ${ }^{15}$ lipid entering the cytosolic storage pool in the postprandial state does offer a potential site for the regulation of lipid appearance into the circulation.

The aim of this study has been to attempt to visualise the "enteral" lipid storage pool responsible for the well documented second meal effect for plasma lipids, in vivo and in humans. By combining our established two meal protocol ${ }^{3}$ with gastrointestinal biopsies, we sought to investigate lipid storage in jejunal tissue in parallel with metabolic effects. This has increasing significance as raised levels of chylomicrons and chylomicron remnants, which circulate following a meal, are suggested to play a role in the pathogenesis of atherosclerosis. ${ }^{16}$

\section{MATERIALS AND METHODS \\ Patients}

Ten patients (six females, mean age 36.7 years (range 21-55); mean weight $57.8 \mathrm{~kg}$ ) undergoing diagnostic oesophagogastro-duodenoscopy (OGD) at the John Radcliffe Hospital, Oxford, were recruited into the study. All patients were free from concomitant cardiovascular, pulmonary, and renal disease, and exhibited no symptoms of fat malabsorption.

Abbreviations: TG, triacylglycerol; ER, endoplasmic reticulum; apo B48, apolipoprotein B48; MTP, microsomal triglyceride transfer protein; OGD, oesophago-gastro-duodenoscopy; Sf, Svedberg flotation rate; CV coefficient of variation; VLDL, very low density lipoprotein; DGAT, acyl-CoA:diacylglycerol acyltransferase. 
Table 1 Electron microscopy samples of jejunal mucosa from healthy subjects graded for lipid content after having ingested either water or a glucose solution five hours after a $50 \mathrm{~g}$ fat load

\begin{tabular}{lll}
\hline & $\begin{array}{ll}100 \mathrm{ml} \text { water } \\
(\mathrm{n}=5)\end{array}$ & $\begin{array}{l}38 \mathrm{~g} \text { glucose in } \\
100 \mathrm{ml} \text { water } \\
(\mathrm{n}=5)\end{array}$ \\
\hline Grade of biopsy (O-IV) & ++++ & + \\
& ++ & 0 \\
++ & 0 \\
& ++ & 0 \\
& + & 0 \\
Mean grade for group & ++ & 0 \\
\hline
\end{tabular}

Informed written consent was obtained and the study was approved by the Oxfordshire Clinical Research Ethics Committee.

\section{Study design}

The study design was based on an established protocol ${ }^{2}$ in which a high fat breakfast is followed five hours later by a "second meal". Small volumes of water and glucose solution were chosen as "second meal" negative and positive controls, respectively, due to the experimental constraints of performing an OGD only one hour after nutrient ingestion. Patients were randomised into two groups to consume either a small glass of water $(100 \mathrm{ml})$ or a glucose drink $(38 \mathrm{~g}$ of glucose in $100 \mathrm{ml}$ water) five hours following a high fat load. The two treatments will be refereed to as "fat load-water" and "fat load-glucose" and the groups as the "water group" and the "glucose group".

After an overnight fast, an oral fat load (50 g liquid fat: Calogen; SHS, Merseyside, UK) was given to all patients at 07:00 on the morning of their endoscopy. At 12:00, patients received their second nutrient stimulus (glucose or water). OGD was performed at 13:00 (six hours after the oral fat load and one hour after the glucose or water) using a paediatric colonoscope which allowed intubation and biopsy of the jejunum. Frozen sections were processed with oil red-O to identify neutral fat. Histological preparations stained with oil red-O were quantified for lipid content using a semiautomatic analysis system (IBAS, Kontron, Germany) with 5-10 fields of mucosal and submucosal tissue included. Tissue for electron microscopy was fixed with $4 \%$ glutaraldehyde. Samples were post-fixed in $1 \%$ osmium tetroxide in a $0.1 \mathrm{M}$ phosphate buffer, dehydrated in ethanol, followed by treatment with propylene oxide prior to embedding in Spurr's epoxy resin. Thin sections were cut and stained with uranyl acetate and lead citrate prior to examination with a JEOL 1200EX microscope. Between six and 10 electron micrographs were taken at magnifications ranging from $\times 5700$ to $\times 23250$ from a single biopsy in each patient. Samples were examined in a blinded fashion and graded independently from 0 to ++++ for lipid content present within the sections.

\section{Metabolic study}

As it was not possible to take blood samples during OGD, a subgroup of five patients (two females) from the original biopsy group underwent parallel metabolic investigations. This was a randomised crossover design with patients undergoing both protocols (fat load-water; fat load-glucose) on separate days two weeks apart.

After an overnight fast, patients attended the metabolic research unit and an indwelling cannula was inserted into the antecubital vein of the forearm and two fasting blood samples were taken. The $50 \mathrm{~g}$ of liquid fat was given at time $=0$ with blood samples taken at hourly intervals. At $\mathrm{t}=300$ minutes, the second drink of either water or glucose solution was given and blood samples were collected every 15 minutes for a further 90 minutes.

Blood samples were collected into heparinised tubes (Sarstedt, Leicester UK) for analysis of plasma glucose, insulin, and TG, and chylomicron rich fraction (Svedberg flotation rate (Sf) >400) TG and apo B48 concentrations. A preservative was added to plasma to prevent proteolytic degradation of apo B48 (EDTA $(0.5 \mathrm{ml} / \mathrm{l})$, phenylmethylsulphonyl fluoride ( $10 \mathrm{mmol} / \mathrm{l}$ dissolved in isopropanol) and aprotinin ( $10000 \mathrm{MIE} / \mathrm{l}))$. The $\mathrm{Sf}>400$ lipoprotein fraction was isolated by density gradient ultracentrifugation. ${ }^{17}$ Briefly, the density of plasma was adjusted to $\mathrm{d}=1.10 \mathrm{~kg} / \mathrm{l}$ with addition of $\mathrm{d}=1.42 \mathrm{~kg} / \mathrm{l} \mathrm{KBr}$ and then overlayered with $\mathrm{d}=1.063,1.020$,
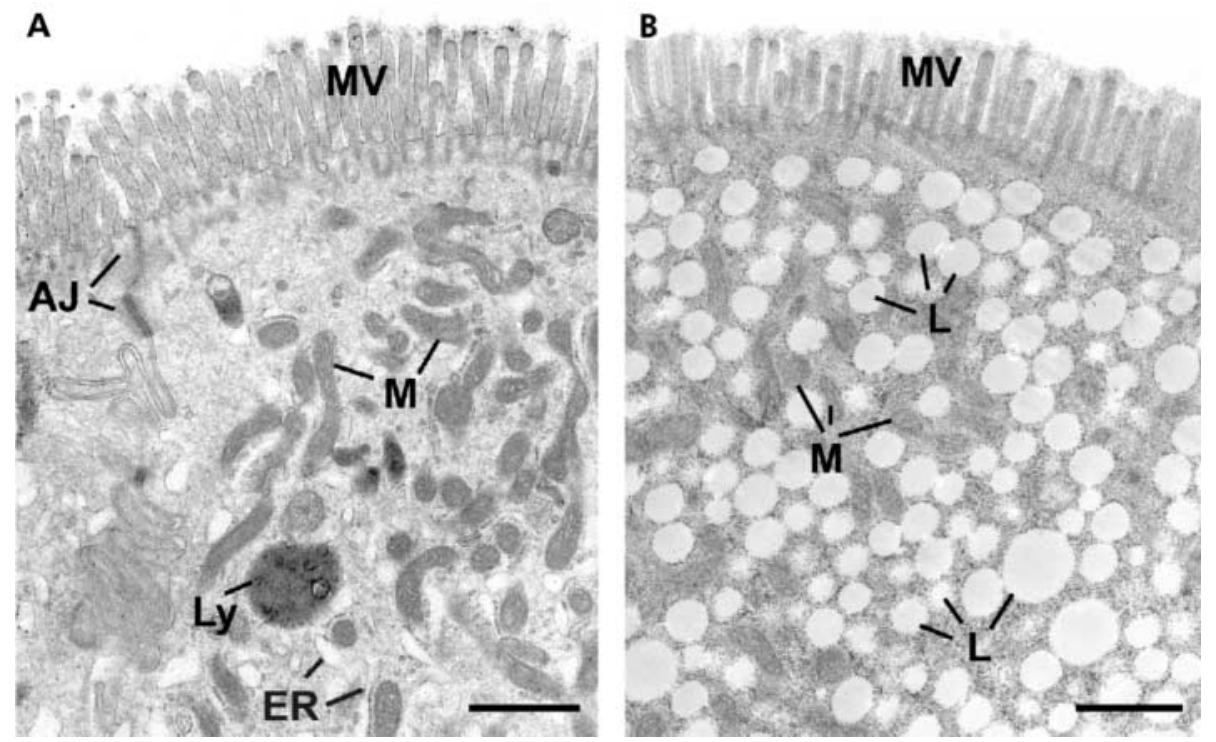

Figure 1 (A) Electron micrograph of jejunal tissue from a female patient one hour after oral glucose ingestion and six hours after a $50 \mathrm{~g}$ fat load. The microvilli (MV) and apical juctional complex (A) denotes the apical surface of the enterocyte. Numerous mitochondria (M), strands of rough endoplasmic reticulum (ER), and a lysosome (Ly) are visible. This represents grade 0 lipid storage. Bar is $1 \mu \mathrm{m}$. (B) Electron micrograph of jejunal tissue from a female patient one hour after oral water ingestion and six hours after a $50 \mathrm{~g}$ fat load. Large numbers of chylomicron sized lipid droplets (L) are present in the apical cytoplasm identified by the presence of microvilli (MV). Lipid is present in both the apical and basal regions of the cell. This represents grade ++++ lipid storage. $M$, mitochondrion. Bar is $1 \mu \mathrm{m}$. 


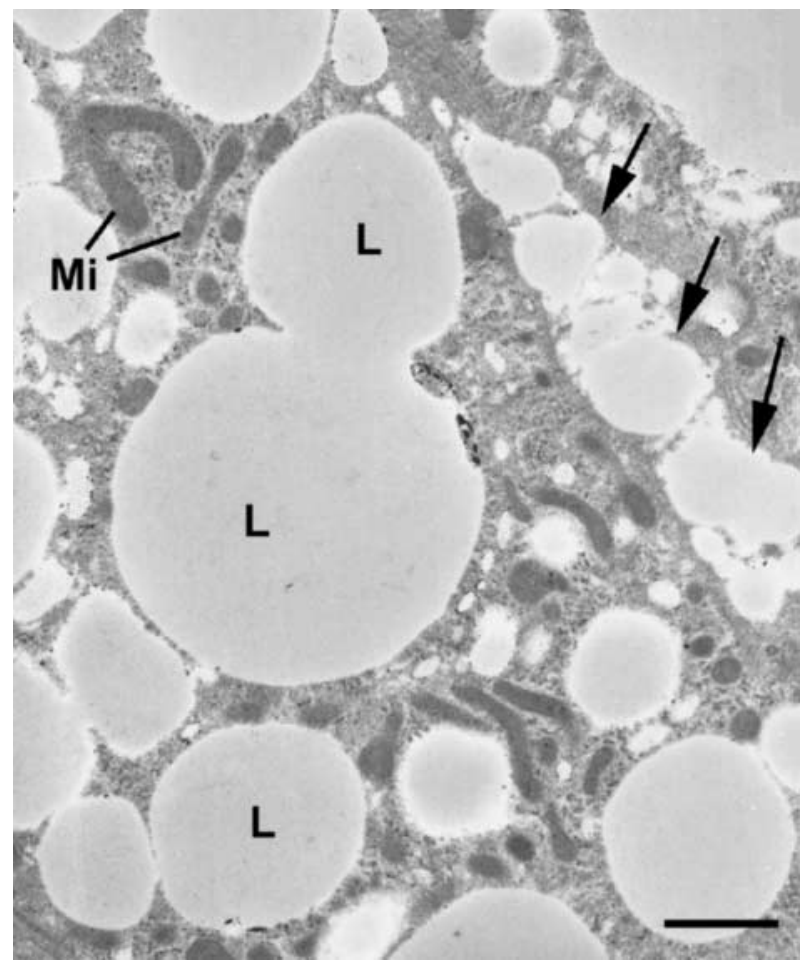

Figure 2 Electron micrograph of jejunal tissue from a male patient one hour after oral water and six hours after a $50 \mathrm{~g}$ fat load. Part of an enterocyte showing large lipid droplets $(L)$ in the basolateral cytoplasm. The droplets are free within the cytosol and not enclosed by a unit membrane. Lipid is also visible within the intercellular space (arrows). Mi, mitochondrion. This represents ++++ lipid storage. Bar is $1 \mu \mathrm{m}$.

and $1.006 \mathrm{~kg} / \mathrm{l} \mathrm{NaCl}$ solutions. Ultracentrifugation was performed at $40000 \mathrm{rpm}(202000 \mathrm{~g})$ for 32 minutes in a SW 40Ti swinging bucket rotor (Beckman Instruments, High Wycombe, UK) at $15^{\circ} \mathrm{C}$. The top $1 \mathrm{ml}$ of the gradient ( $\mathrm{Sf}>400$ fraction) was carefully isolated. Apo B48 was measured using a specific ELISA ${ }^{18}$ with a few modifications. A heptapeptidethyroglobulin conjugate consisting of the terminal residues of the apo B48 molecule was used as the coating material in the ELISA format. Samples were incubated with a specific polyclonal anti-apo B48 antibody, which recognises the C terminal region of the protein on the surface of lipoprotein particles and does not show cross reactivity for apo B100. The intra-assay coefficient of variation (CV) was $4.5 \%$. Plasma glucose concentrations were measured enzymatically using an IL Monarch automated analyser (Instrumentation Laboratory, Warrington UK). Insulin was measured by radioimmunoassay using a commercially available kit (Pharmacia and UpJohn, Milton Keynes, UK) exhibiting both inter- and intra-assay coefficients of variation of less than $10 \%$. TG concentrations in plasma and $(\mathrm{Sf}>400)$ lipoprotein fraction were measured using an enzyme based colorimetric kit (Instrumentation Laboratory). The intra-assay CV was $1.7 \%$.

\section{Statistics}

Data were analysed using SPSS version 10 (SPSS inc.; Chicago, Illinois, USA). Plasma results presented in time courses are mean (SEM) values. Postprandial data were analysed using repeated measures ANOVA. Summary data such as areas under the curve were calculated by the trapezoid method and analysed by paired Student's $t$ test. Biopsy data (grade of electron microscopy samples and quantitative staining with oil red-O) were analysed by one way ANOVA for unpaired samples. A p value of $<0.05$ was taken as the level of significance.
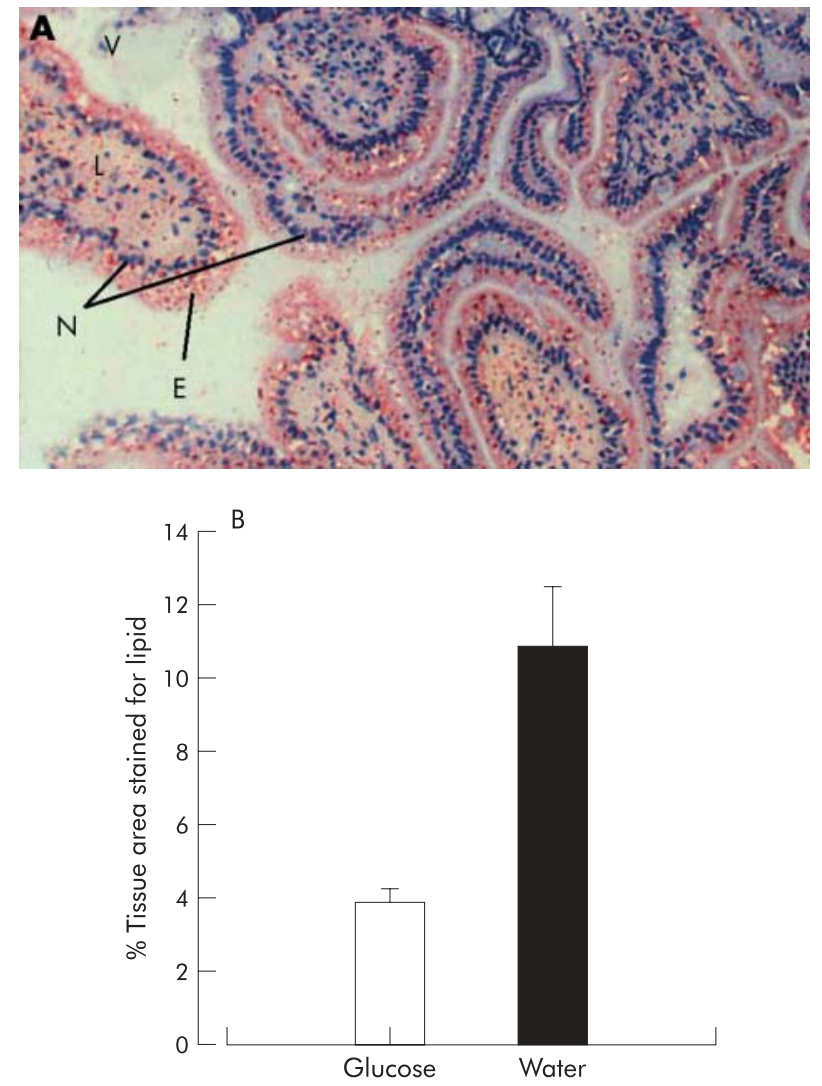

Figure 3 (A) Light microscopy image $(\times 100)$ of jejunal mucosa and submucosa in a female patient one hour after water ingestion and six hours after a $50 \mathrm{~g}$ fat load. Neutral lipid is stained red with oil red-O. Cells of the villus tip (V) are clearly visible. Nuclei (N) of individual enterocytes (E) are stained black. Staining for fat is present in both the enterocytes and the lamina propria (L). (B) Percentage of total tissue stained for fat with oil red-O in patients one hour after either water or glucose ingestion and six hours after a $50 \mathrm{~g}$ fat load. Mean (SEM), $\mathrm{n}=5$. There was a significant difference in the total staining for fat between the two treatment groups, $\mathrm{p}=0.032$ (Mann-Whitney test).

\section{RESULTS}

During endoscopic examination, all 10 patients were found to have normal jejunal mucosa $(2 \times$ lactose intolerant; $4 \times$ irritable bowel syndrome; $2 \times$ non-ulcer dyspepsia; $1 \times$ oesophagitis). There was no evidence of liquid fat emulsion remaining within the lumen of the stomach, duodenum, or jejunum six hours after the oral fat load in either treatment group.

\section{Biopsy study}

Table 1 displays the grade of fat deposit present within the jejunal biopsy sample. Six hours after a fat load there were numerous lipid vacuoles of varying size within the cytoplasm of the enterocytes for $6 / 10$ patients ( $1 / 5$ fat load-glucose (fig 1A); $5 / 5$ fat load-water; $p=0.005$ (fig lB)). The vacuoles were not limited by a "unit membrane" which distinguishes the lipid structures from other cell organelles. It is likely that there were some deposits of phospholipids forming a limiting layer but we were unable to detect these using our histological methods. The lipid droplets ranged in size from approximately $0.5 \mu \mathrm{m}$ to $6 \mu \mathrm{m}$, with significant variation both within and between subjects. These were not confined to the basolateral region of the cell and did not appear to be associated with the secretory apparatus (ER or Golgi) which was clearly visible following electron microscopy ( fig 2). Lipid was also evident in the interstitium between adjacent enterocytes. On light microscopy, jejunal samples from the water group exhibited significantly more lipid staining than did samples from the 


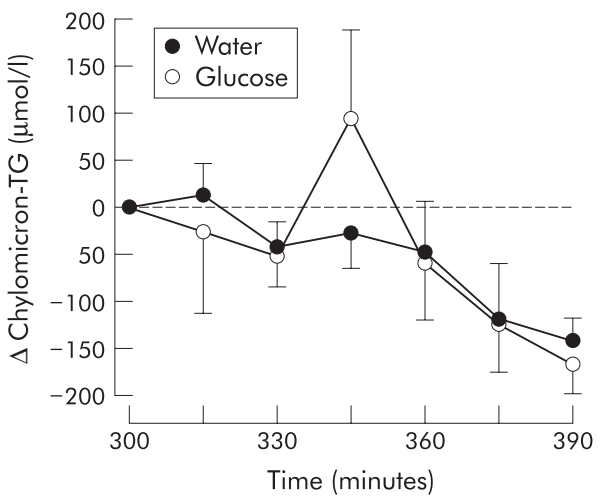

Figure 4 Change in chylomicron-triacylglycerol (TG) concentration following either water or glucose ingestion at $\mathrm{t}=300$ minutes. A $50 \mathrm{~g}$ fat load was administered to all patients at $t=0$. Mean $(S E M)$ values are displayed, $n=5$. Repeated measure ANOVA showed a significant difference between the two treatments $(p=0.033)$.

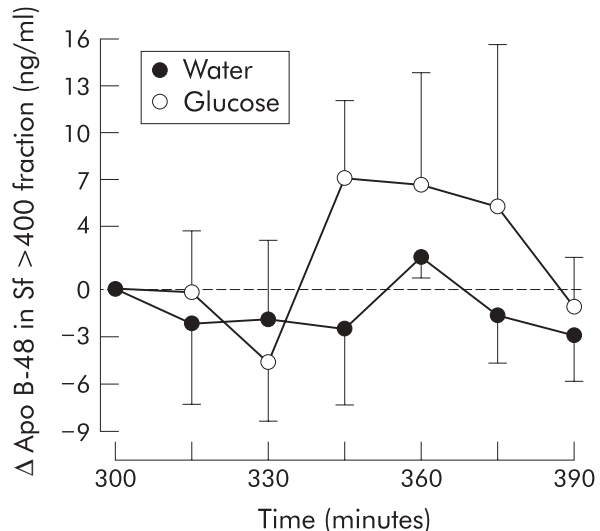

Figure 5 Change in apolipoprotein (Apo) B48 concentration within the chylomicron rich fraction following either water or glucose ingestion at $t=300$ minutes. A $50 \mathrm{~g}$ fat load was administered at $t=0$. Mean $(S E M)$ values are displayed, $n=5$. The integrated response (IAUC) was significantly higher following oral glucose (paired Student's $t$ test, $\mathrm{p}=0.041$ ).

glucose group. Lipid was evident within both the enterocytes and the lamina propria of the villus tip (fig 3A).

\section{Metabolic study}

As the metabolic load was identical in both groups for the first 300 minutes of the study, data are only presented for 300-390 minutes (90 minutes after the second stimulus).

The plasma chylomicron-TG ( $\mathrm{Sf}>400$ ) response following water or glucose ingestion is shown in fig 4 . There was a significant peak in chylomicron-TG 45 minutes after glucose ingestion which was not evident in the water group $(\mathrm{p}=0.033)$. A similar pattern was seen for plasma TG concentration, with a peak observed 45 minutes following glucose ingestion but not with oral water $(p=0.008$ ) (data not shown). Apo B48 concentrations within the chylomicron rich fraction (Sf >400) were also significantly higher in the glucose group, with a peak again reached by 45 minutes post ingestion (fig 5). The TG/apo B48 ratio in the chylomicron rich fraction in the first 300 minutes of the study (after fat ingestion alone) was significantly lower than that obtained (300-390 minutes) following glucose ingestion (10.6 $v 15.6$ mmol TG per $\mu$ g apo B48; $\mathrm{p}=0.043$ ).

As expected, the plasma glucose concentration rose with a peak at 45 minutes after oral glucose ingestion whereas there was no effect of oral water. There was an increase in the plasma insulin concentration which remained elevated for the
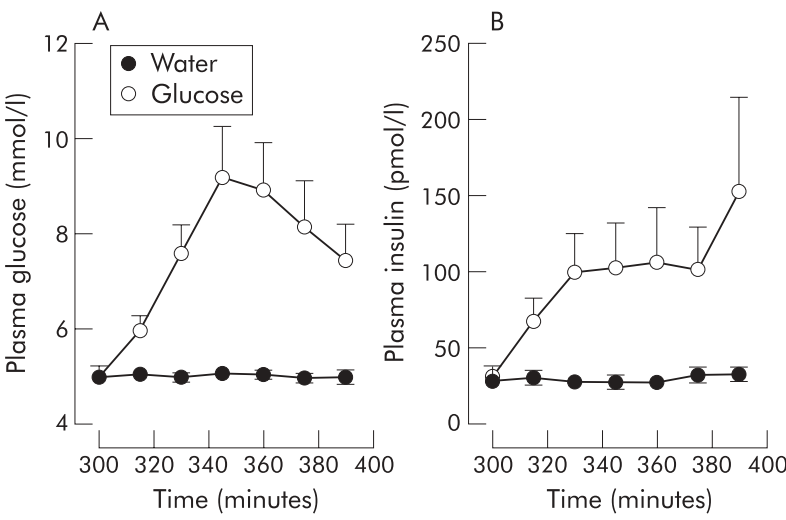

Figure 6 Plasma glucose (A) and insulin (B) concentrations following either water or glucose ingestion at $\mathrm{t}=300$ minutes. A $50 \mathrm{~g}$ fat load was administered at $t=0$. Mean (SEM), $n=5$. Repeated measures ANOVA showed a significant difference between the two treatments for both plasma glucose $(p=0.015)$ and insulin $(p<0.001)$ concentrations.

remainder of the study despite a drop in plasma glucose concentration (fig 6).

\section{DISCUSSION}

The combination of metabolic data with electron microscopy images has allowed demonstration for the first time that during the second meal effect for plasma TG, the cytosolic storage pool of lipid contained within the jejunal enterocytes is significantly reduced. Six hours after the high fat load there were significant amounts of lipid visible within the enterocytes, the intercellular spaces, and the lamina propria of the villus tip. The lipid visible within the jejunal tissue was significantly reduced one hour after oral glucose, coinciding in the parallel metabolic studies with a peak in endogenous lipid particles in the plasma. The second meal effect appears to reflect both an increase in the mobilisation of cytosolic TG and an increase in the movement of these lipid particles through the secretory pathway and from lymph to circulation; an effect which we have shown may be independent of further fat ingestion.

The large lipid droplets (up to $6 \mu \mathrm{m}$ ) observed in this study and the lack of integrity with the ER membrane led to the conclusion that following fat absorption, re-esterified fat is stored within the cytoplasm. This adds credence to the theory that the transfer of TG from the ER into the secretory pathway can become saturated, resulting in storage pools of TG within the cytosol. ${ }^{819}$ Chylomicron assembly is similar to the assembly of very low density lipoprotein (VLDL) particles in the liver. In hepatocytes, it is known that a proportion of the secreted TG as VLDL-TG is derived from this cytosolic pool ${ }^{20}$; that the same phenomenon should occur within enterocytes is not perhaps surprising, although until now difficult to demonstrate in vivo and in humans.

For the stored TG to be made available, it must undergo hydrolysis within the cytosol followed by re-esterification in the ER. It has been shown recently that rat enterocytes express pancreatic TG lipase ${ }^{21}$ and in Caco-2 cells the majority of this lipase activity is found in the cytosolic fraction. ${ }^{22}$ Addition of exogenous lipase to the apical surface of Caco-2 cells results in both increased fat hydrolysis and enhanced basolateral lipid secretion, ${ }^{22}$ an observation which would explain our in vivo second meal effect. The relationship between oral glucose and cytosolic lipase concentration in this study may be modulated via the concomitant increase in circulating plasma insulin. Increased TG hydrolysis has been demonstrated in hepatocytes cultured with insulin ${ }^{20}$ although the effect of circulating insulin on cytosolic lipase within enterocytes is currently 
unknown. In Caco-2 cells, short term incubation with insulin also increases intestinal fatty acid binding protein levels ${ }^{23}$ which may be important for the movement of these newly formed products of lipolysis to the ER. The potential for the rate of chylomicron release from the enterocyte to be "controlled" by the ER itself cannot be disregarded. Recent studies in mice lacking the enzyme acyl-CoA:diacylglycerol acyltransferase 1 (DGAT1), which catalyses the final step in TG synthesis, have revealed large lipid droplets within the cytoplasm of enterocytes. ${ }^{24}$ DGAT activity in the heart ${ }^{25}$ and adipocyte ${ }^{26}$ has been shown to increase in animal models of diabetes although whether this is related to increased circulating levels of glucose per se remains to be determined.

Following exocytosis from the basolateral membrane, the newly secreted chylomicron particles first enter the intercellular space before migrating via the lacteals into the circulation; however, a proportion of fat is retained in the lymphatics. ${ }^{27}$ Following oral glucose, there was significantly less fat present within the lamina propria compared with that following water ingestion, indicating that chylomicrons that had been retained in the lymph following the original high fat load also contributed to the observed second postprandial peak in plasma lipids. Factors affecting the rate of movement of preformed chylomicrons through the lymph are therefore likely to be important in determining the magnitude of the second meal effect. Glucose in the luminal fluid has been shown to stimulate both fluid uptake and lymph flow, ${ }^{28}$ increasing sympathetic activity ${ }^{29}$ leading to vasodilation of the submucosa arterioles and enhanced intestinal blood flow..$^{30}$ Insulin also leads, directly or indirectly, to vasodilation in muscle ${ }^{29}$ and adipose tissue. ${ }^{31}$

Apo B48, synthesised by bound ribosomes in the ER, is the major structural protein component and is essential for the formation and secretion of chylomicrons. Following oral glucose, the chylomicron apoB48/TG ratio differed significantly from the ratio following the earlier fat load which may potentially be interpreted as a difference in the composition of the secreted lipoproteins between the original fat load and the glucose load. The second meal effect increased the chylomicron TG/apo B48 ratio, indicating the secretion of larger particles, as each chylomicron is known to contain only a single apo B48 particle. ${ }^{11}$ Addition of lipid to the newly formed apo B48 to form the lipoprotein particle occurs in the lumen of the ER/Golgi and is mediated by MTP, and so regulation of MTP activity is likely to be important in determining particle size. Inhibition of MTP in Caco-2 cells has been shown to reduce the amount of TG per particle ${ }^{32}$ and conversely increases in MTP result in the secretion of larger particles. ${ }^{33}$ Whether there is acute regulation of MTP by either glucose or insulin has yet to be demonstrated.

This secondary release of lipids from the "enteral" store has been studied metabolically for many years ${ }^{1-3}$ without knowing the site of this "enteral" store. There is no reported difference in the magnitude of the second meal effect between low and high fat meals; nothing is known on the effects of individual amino acids on this process although from our metabolic studies carbohydrate content would appear to be important. ${ }^{2}$ In this study, we demonstrated an almost complete mobilisation of all of the stored cytosolic TG following oral glucose $(4 / 5$ subjects showed no fat storage following glucose ingestion) with only minimal amounts of fat still visible within the lamina propria.

In conclusion, following a high fat meal in humans, a proportion of the fat load remains both within the enterocytes as neutral lipid droplets contained within the cytosol and as preformed chylomicrons retained within the lymph. When glucose is ingested as a second meal, TG mobilisation within the enterocyte is stimulated, producing new chylomicron particles which are exported from the cell. A concomitant increase in lymph flow results in movement of chylomicron particles, resulting in a rapid peak in plasma TG. In this study we have made significant progress in locating the storage pool of dietary TG responsible for the second meal effect in vivo in humans; however, further work is necessary, potentially utilising cultured human enterocytes, in determining the precise mechanisms involved.

\section{ACKNOWLEDGEMENTS}

This study was funded by the Biotechnology and Biological Sciences Research Council (UK).

\section{Authors' affiliations}

M D Robertson, K N Frayn, Metabolic Research Group, Oxford Centre for Diabetes, Endocrinology, and Metabolism, University of Oxford, Oxford, UK

M Parkes, D P Jewell, Nuffield Department of Gastroenterology, University of Oxford, Oxford, UK

B F Warren, D J P Ferguson, Department of Pathology, John Radcliffe Hospital, Oxford, UK

K G Jackson, Hugh Sinclair Unit for Human Nutrition, School of Food Biosciences, University of Reading, Reading, UK

\section{REFERENCES}

1 Cohn JS, McNamara JR, Krasinski SD, et al. Role of triglyceride-rich lipoproteins from the liver and intestine in the etiology of postprandial peaks in plasma triglyceride concentration. Metabolism 1989:38:484-90

2 Evans K, Kuusela PJ, Cruz ML, et al. Rapid chylomicron appearance following sequential meals: Effects of second meal composition. Br J Nutr 1998;79:425-9.

3 Fielding BA, Callow J, Owen RM, et al. Postprandial lipaemia: The origin of an early peak studied by specific fatty acid intake during sequential meals. Am J Clin Nutr 1996;63:36-41.

4 Williams CM, Moore F, Morgan L, et al. Effects of $n-3$ fatty acids on postpramdial triacylglycerol and hormone concentrations in normal subjects. Br J Nutr 1992;68:655-66.

5 Jackson KG, Robertson MD, Fielding BA, et al. Second meal effect: modified sham feeding does not provoke the release of stored triacylglycerol from a previous high-fat meal. Br J Nutr 2001;85:149-56

6 Emken EA, Rohwedder WK, Adlof RO, et al. Absorption and distribution of deuterium-labelled trans- and cis-octadecenoic acid in human plasma and lipoprotein lipids. Lipids 1986;21:589-95.

7 Tso P, Balint JA. Formation and transport of chylomicrons by enterocytes to the lymphatics. Am J Physiol 1986:250:G715-26.

8 Cartwright IJ, Higgins JA. Increased dietary triacylglycerol markedly enhances the ability of isolated rabbit enterocytes to secrete chylomicrons: an effect related to dietary fatty acid composition. J Lipid Res 1999:40: 1858-66.

9 Benoist F, Grand-Perret T. Apo B-100 secretion by HepG2 cells is regulated by the rate of triglyceride biosynthesis but not by intracellular lipid pools. Arterioscler Thromb Vasc Biol 1996;16:1229-35.

10 Hamilton RL, Wong JL, Chem CM, et al. Chylomicron-sized particles are formed in the setting of apolipoprotein B deficiency. J Lipid Res 1998;39: 1543-57

11 White DA, Bennett AJ, Billett MA, et al. The assembly of troiacylglycerol-rich lipoproteins: an essential role for the microsomal triacylglycerol transfer protein. Br I Nutr 1998:80:219-29.

12 Vernier JM, Sire MF. Is the Golgi apparatus the obligatory final step for lipoprotein secretion by intestinal cells? Tissue Cell 1986;18:447-60.

13 Tytgat GN, Rubin CE, Saunders DR. Synthesis and transport of lipoprotein particles by intestinal absorptive cells in man. J Clin Invest 1971;50:2065-78

14 Mansbach CM, Nevin P. Intracellular movement of triacylglycerols in the intestine. J Lipid Res 1998;39:963-8.

15 Yang LY, Kuksis A, Myher JJ. Similarities in surface lipids of chylomicrons from glyceryl and alkyl ester feeding: major components. Lipids 1991;26:806-18

16 Zilversmit DB. Atherogenesis: a postprandial phenomenon. Circulation 1979;60:473-85.

17 Karpe F, Hamsten A. Determination of apolipoproteins B-48 and B-100 in triglyceride-rich lipoproteins by analytical SDS-PAGE. J Lipid Res 1994;35:1311-17.

18 Lovegrove JA, Isherwood SG, Jackson KG, et al. Quantitation od apolipoprotein B48 in triacylglycerol-rich lipoproteins by a specific linked immunosorbent assay. Biochim Biophys Acta 1996;1301:221-9.

19 Atkinson D, Small DM. Recombinant lipoproteins: implications for structure and assembly of native lipoproteins. Annu Rev Biophys Biophys Chem 1986;15:403-56.

20 Gibbons GF, Wiggins D. Intracellular triacylglycerol lipase: its role in the assembly of hepatic very low density lipoprotein (VLDL). Adv Enzyme Regul 1995;35: 179-98.

21 Mahan JT, Heda GD, Rao RH, et al. The intestine expresses pancreatic triacylglycerol lipase: regulation by dietary lipid. Am J Physiol Gastrointest Liver Physiol 2002;280: G 1 187-96.

22 Spalinger J, Seidman E, Menard D, et al. Endogenous lipase activity in caco-2 cells. Biochim Biophys Acta 1998;1393:1 19-27. 
23 Dube N, Delvin E, Yotov W, et al. Modulation of intestinal and liver fatty acid- binding proteins in caco- 2 cells by lipids hormones and cytokines. $J$ Cell Biochem 2001;81:613-20.

24 Buhman KK, Smith SJ, Stone SJ, et al. DGAT1 is not essential for intestinal absorption or chylomicron synthesis. J Biol Chem 2002;277:25474-9.

25 Schoonderwoerd K, Broekhoven-Schokker S, Hulsmann WC, et al. Properties of phospatidate phosphohydralase and diacylglycerol acyltransferase activities in isolated rat heart: Effect of glucagon, ischaemia and diabetes. Biochem J 1990;268:487-92.

26 Baht HS, Saggerson ED. Comparison of triacylglycerol synthesis in rat brown and white adipocytes. Effects of hypothyroidism, strepozotocin diabetes on enzyme activities and metabolic fluxes. Biochem $J$ 1988;250:325-33.

27 Mendeloff Al. The effects of eating and of sham feeding upon the absorption of vitamin A palmitate in man. J Clin Invest 1954:33:1015-21.
28 Lee JS. Luminal and plasma glucose concentrations on intestinal fluid absorption and lymph flow. Am J Physiol 1987;252:G568-73.

29 Scott EM, Greenwood JP, Vacca G, et al. Carbohydrate ingestion, with transient endogenous insulinaemia produces both sympathetic activation and vasodilation in normal humans. Clin Sci 2002;102:523-9.

30 Bohlen HG, Unthank JL. Rat intestinal lymph osmolarity during glucose and oleic acid absorption. Am J Physiol 1989;257:G438-46.

31 Karpe F, Fielding BA, Ardilouze JL, et al. Effects of insulin on adipose tissue blood low in man. J Physiol 2002;540:1087-93.

32 Van Greevenbroek MMJ, de Bruin TWA. Chylomicron synthesis by intestinal cells in vitro and in vivo. Atherosclerosis 1998;141(suppl 1):S9-16.

33 Haidari $M$, Leung N, Mahbub F, et al. Fasting and postprandial overproduction of intestinally derived lipoproteins in an animal model of insulin resistance: evidence that chronic fructose feeding in hamsters accompanied by enhanced intestinal de novo lipogenesis and apoB48-containing lipoprotein production. J Biol Chem 2002; 277:31646-55. 